\title{
Effect of annealing treatments on the anisotropy of a magnesium alloy sheet processed by severe rolling
}

\author{
J. A. del Valle and O. A. Ruano \\ Dept. of Physical Metallurgy, Centro Nacional de Investigaciones Metalúrgicas (CENIM), \\ CSIC. Avda. de Gregorio del Amo, 8, 28040 Madrid, Spain
}

Corresponding author: J. A. del Valle

e-mail: delvalle@cenim.csic.es

Tef: (+34) 915538900

Fax: (+34) 915347425

\begin{abstract}
The effect of annealing treatments on the normal plastic anisotropy ( $r$-value) of a magnesium alloy, AZ61, processed by severe rolling was investigated. The various annealing treatments produce two effects on microstructure: grain coarsening and slight weakening of the texture. In addition, these treatments produce a noticeable decrease of the anisotropy that was correlated with an increase in strain rate sensitivity and a decrease of work hardening rate. It is concluded that an enhanced contribution of basal slip occurs as a consequence of the annealing treatments.
\end{abstract}

Keywords: Metals and alloys; Magnesium alloys; Rolling; Texture; Mechanical properties; Plastic anisotropy.

\section{Introduction}

In previous works, tensile tests at room temperature for AZ31 and AM60 magnesium alloys have shown a decrease of work hardening (WH) $[1,2,3,4]$ and an increase of strain rate 
sensitivity (SRS) with decreasing grain size $[3,4,5]$. This dependence was especially significant for grain sizes finer than about $15 \mu \mathrm{m}$. In these investigations, the materials were processed in order to obtain a fine grain size. Subsequently, grain growth was induced by means of annealing thermal treatments, and the grain size effect was investigated. Minor texture weakening are usually reported under these treatments $[1,3,5,6]$.

Two alternatives were considered to explain the grain size dependence of the $\mathrm{WH}$ and the SRS. It was speculated with a contribution of grain boundary sliding (GBS) to deformation [1, 3, 5] based on reports of deformation of pure magnesium at room temperature by Hauser et al. [7] and recently by Koike et al [8]. Taking into account that, for grain sizes into the nanometric scale, very low WH rates are observed at room temperature in Mg, together with an increase in the SRS parameter yielding values close to 0.5 (typical of GBS)[4], some kind of transition towards this behavior could be expected for decreasing grain size. Another possible explanation, pointed on changes in the activity of the different slip systems and/or twining systems $[1,2,3,9]$ induced by the grain refinement.

A useful tool for analyzing deformation mechanisms is through the measurement of the normal plastic anisotropy ( $r$-value) [10]. In the present work, a study was made of the evolution of the anisotropy with strain in tensile tests performed in the rolling and transverse directions of AZ61 magnesium alloy. These anisotropy measurements could give a clue on changes in the deformation mechanism and in the relative activity of the operating slip systems.

\section{Materials and methods}

Alloy AZ61 (Mg-6wt\%Al-0.75wt\%Zn) was received in the form of as-extruded plate, 10 $\mathrm{mm}$ in thickness [11]. Slabs of the as-received material were processed by severe hot rolling at $400^{\circ} \mathrm{C}$ using two passes, the first to $7 \mathrm{~mm}$ and the second to $1.8 \mathrm{~mm}$ in thickness. Figure $1 \mathrm{a}$ shows the microstructure obtained. The grain size, $d$, measured by the mean length intercept method with a correction factor of 1.74 , is $3.6 \mu \mathrm{m}$. In addition, annealing treatments were performed, to produce samples with coarser grain sizes as detailed in Table I.

Texture measurements were performed in the back-reflected mode by X-Ray diffraction 
using a X-Ray Bruker diffractometer and $\mathrm{Co}_{\alpha}$ radiation. Pole figures were constructed from the X-Ray diffraction data.

Dog-bone tensile samples of $12 \mathrm{~mm}$ gage length, transversal section of $1.8 \mathrm{x} 4 \mathrm{~mm}^{2}$ were spark-machined with the tensile axis parallel and perpendicular to the rolling direction. These samples were termed $\mathrm{RD}$ and $\mathrm{TD}$ respectively.

Tensile tests were performed at a strain rate of $5 \times 10^{-3} \mathrm{~s}^{-1}$ at room temperature. In order to determine the variation of the width and thickness of the gauge length, tensile tests were interrupted at given strains levels: $0.04,0.08,0.12$, and immediately after the maximum stress point (before the fracture of the sample). Ten reference points were initially marked along the gauge length and, at each strain level, the four sides of the rectangular cross section of the tensile sample were measured using the length measurement tools of a microindenter for each reference point. After collecting the width/thickness data the sample was further reloaded to the following strain level. These data allow calculation of the normal strain anisotropy given by:

$$
r=\frac{\ln \left(\mathrm{w}_{\mathrm{f}} / \mathrm{w}_{\mathrm{o}}\right)}{\ln \left(\mathrm{t}_{\mathrm{f}} / \mathrm{t}_{\mathrm{o}}\right)}
$$

where $\mathrm{w}_{\mathrm{f}}$ and $\mathrm{w}_{\mathrm{o}}$ are the final and initial width respectively, and $\mathrm{t}_{\mathrm{f}}$ and $\mathrm{t}_{\mathrm{o}}$ are the final and initial thickness respectively. The strain corresponding to each reference point was calculated from the variation of local cross-sectional area using:

$$
\varepsilon=\ln \left(\mathrm{A}_{\mathrm{o}} / \mathrm{A}_{\mathrm{f}}\right)=\ln \left(\mathrm{w}_{\mathrm{f}} \mathrm{t}_{\mathrm{f}} / \mathrm{w}_{\mathrm{o}} \mathrm{t}_{\mathrm{o}}\right)
$$

where $A_{f}$ and $A_{o}$ are the final and initial cross-sectional area respectively.

\section{Results}

Figure 2 shows the pole figures corresponding to the three types of tensile samples prepared. As can be seen in the Figure, the AZ61 samples have a basal texture. Minor changes in texture occurred during annealing for the fine grained samples, $\mathrm{d}=3.6$ to $6.6 \mu \mathrm{m}$, and some decrease in intensity and a widening of the basal texture is observed for the $d=36 \mu \mathrm{m}$ sample. The (0002) pole figure shows that the angular tilting is greater towards the rolling direction 
(RD) than towards the transverse direction (TD), as usual in rolled magnesium [12].

Texture measurements, on samples tested up to a tensile strain of 0.15 , shows that the caxis rotates towards the sample normal and away of the tensile direction, due the basal slip activity. The pole figures corresponding to the prismatic planes (10-10) (not shown in Fig. 2) shows that directions $\langle 01 \overline{1} 0$ became parallel to the tensile axis, i.e. a fibre texture is formed during strain.

The stress-strain curves of RD and TD samples are shown in Figure 3a-b and the $r$-data are given in Figures 3c-d. The range of uniform deformation is marked in these last figures by a vertical dotted line. In this range, $r$ is determined as the average over the ten reference points.

\section{Discussion}

Figures 3a-b shows an increase of the proof stress with decreasing grain size as expected by the Hall-Petch relation. Figures 3c-d show that the general trend consist in a monotonous increase of anisotropy with increasing strain for all samples evaluated. This result can be related to an increasing activity of non-basal $<\mathrm{a}>$ slip systems [9]. The effect of the annealing treatments consists in a decrease in the anisotropy which becomes stronger as the severity of annealing treatment increases. This decrease in r-values is approximately independent of strain, that is, the curves are displaced vertically when microstructure is modified. This suggests that the differences in the anisotropy with severity of heat treatment (or increasing grain size) are not a consequence of a different evolution of the slip system activity during deformation. Conversely, these differences apparently are caused by different slip system activity from the beginning of deformation.

For the moderate annealing treatment, $\mathrm{d}=6.6 \mu \mathrm{m}$, a decrease of anisotropy is observed with minor texture changes respect to the as-rolled condition. The samples with the largest grain size of $36 \mu \mathrm{m}$ show the lowest anisotropy values. In this case there is a widening of the basal pole distribution. Furthermore, the anisotropy is considerably larger in TD samples than in RD samples for the same processing condition and same grain size. This is a known texture effect 
$[9,10]$. The tilting of basal planes toward RD favors basal slip in detriment of non-basal $<a>$ prismatic slip when the tension axis is parallel to RD, leading to lower $r$-values in comparison to TD samples.

Jain et al [9] reported $r$-values corresponding to TD samples of AZ31 in the range of 4-5 and for RD samples in the range 2-3 with no meaningful dependence on grain size in the range 13-90 $\mu \mathrm{m}$. These authors find exclusively a slight increase of the r-value for RD samples with increasing grain size which was attributed to subtle changes in texture during annealing treatments used. Yang and Ghosh [3] reported an increase of anisotropy and SRS for AZ31 refined $\mathrm{d} \approx 3 \mu \mathrm{m}$, the evolution of anisotropy with strain was not measured.

Our results show a reduction in anisotropy, related to a progressive enhancement of basal slip, as the severity of the annealing treatments increases, i.e. with increasing grain size. Two microstructural features could cause these variations in the slip system activity:

i) Basal slip could be enhanced by the weakening in texture caused by the severity of the annealing treatments (and not by an increase in grain size) increasing the tilting of the basal planes, away from the normal direction. In this work only slight texture variations between samples with $\mathrm{d}=3.6$ and $6.6 \mu \mathrm{m}$ was observed. It is unlikely that this effect may explain the noticeable decrease in the r-value in TD direction. However, it cannot be disregarded that very slight texture changes could produce considerable changes in slip activity. Modelling using polycrystal plasticity simulations is being carried out to clarify this question.

ii) Prismatic slip should increase with decreasing values of the ratio $\tau_{\text {prism }} / \tau_{\text {basal }}$, of the critical resolved shear stresses for slip in the prismatic and in basal planes [10]. Therefore, any alteration of this ratio could lead to changes in anisotropy. For example, Jain et al [9] and Proust et al [13] speculated with the possibility of having different Hall-Petch relations for different slip systems. In that case, the ratio $\tau_{\text {prism }} / \tau_{\text {basal }}$ could be grain size dependent and consequently the $r$-value. This hypothesis is in agreement with our previous statement that the differences in anisotropy are caused by the different slip system activity from the beginning of deformation.

It is worth noting that the present results are not compatible with the operation of GBS. 
Clearly, if a fine grain size enhances GBS, a decrease of anisotropy with decreasing grain size is expected since this mechanism is not affected by texture [14] which is contrary to the experimental results.

Previous work on AZ31 alloy showed a grain size dependence of SRS and WH [1,5]. The same dependence has been observed in AZ61 alloy and will be the subject of a future publication. In the light of these results, the decrease of the WH rate and the increase of SRS, as grain size is refined, appears to be necessary linked to the increase in anisotropy and has to be rationalized in terms of a increasing amount of prismatic slip, being the rate controlling mechanism according to Armstrong [15].

\section{Conclusions}

The evolution of the normal anisotropy with the strain of processed and annealed samples of a magnesium alloy, AZ61, was investigated. The experimental results show that these thermal treatments produce a noticeable decrease of plastic anisotropy with simultaneous grain size coarsening and slight changes in the basal texture. It is concluded that an enhanced contribution of basal slip occurs from the beginning of tensile strain in the annealed samples. These results can be correlated with previous results on increasing SRS and decreasing WH. Two alternatives are proposed to explain the enhancement of basal slip: subtle changes in the initial texture during annealing and/or a grain size effect on the ratio $\tau_{\text {prism }} / \tau_{\text {basal }}$.

\section{Acknowledgements}

The authors acknowledge financial support from the CICYT grant MAT 2006-02672 awarded by the Spanish Ministry of Education and Science. 


\begin{tabular}{|l|c|}
\hline \multicolumn{1}{|c|}{ TMP } & Grain size \\
\hline Hot rolled $400^{\circ} \mathrm{C}$, two passes & $3.6 \mu \mathrm{m}$, Fig. 1(a) \\
Hot rolled + annealing $300^{\circ} \mathrm{C}, 100 \mathrm{~min}$. & $6.6 \mu \mathrm{m}$ \\
Hot rolled + annealing $400^{\circ} \mathrm{C}, 1000 \mathrm{~min}$. & $36 \mu \mathrm{m}$, Fig. 1(b) \\
\hline
\end{tabular}

Table I. Thermomechanical processing routes used for controlling grain size.

Figures
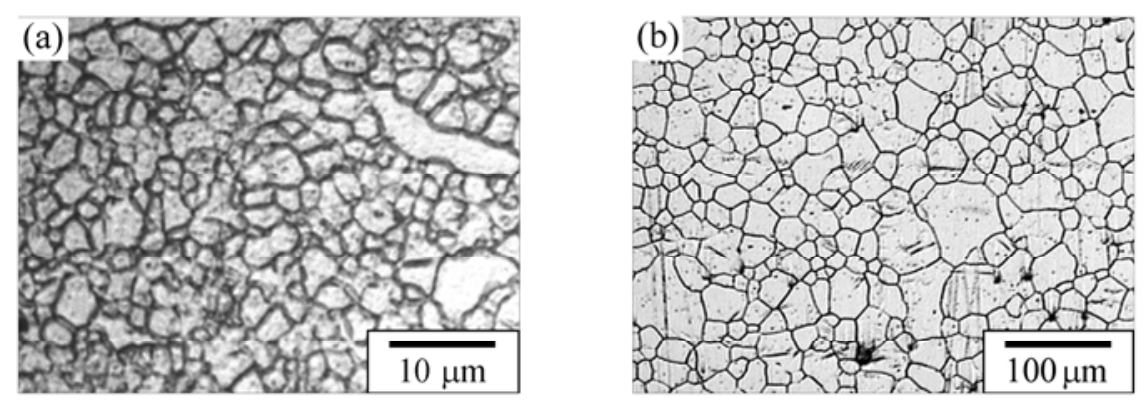

Figure 1. Optical micrographs showing the microstructure of the AZ61 alloy after the processing routes given in Table I.

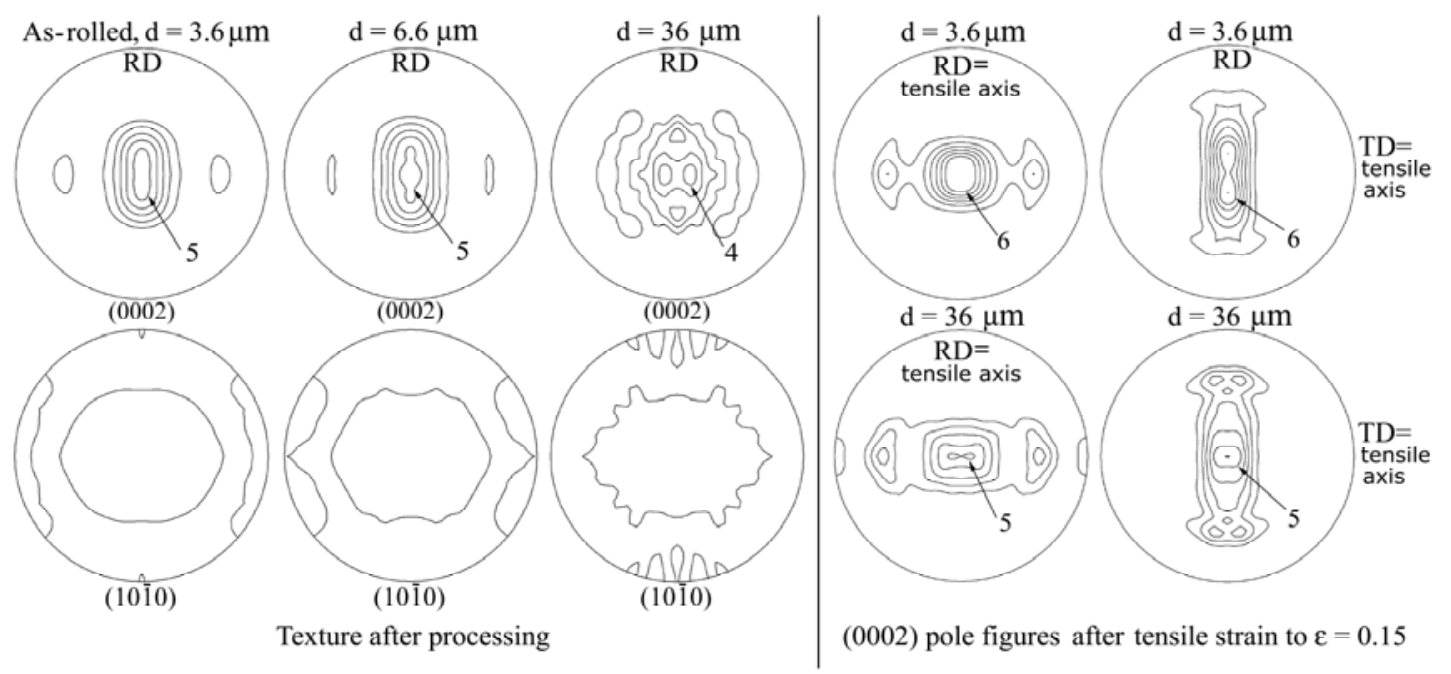

Figure 2. Pole figures of the AZ61 alloy after the processing routes given in Table I, and after 0.15 tensile strain in RD and TD directions. 

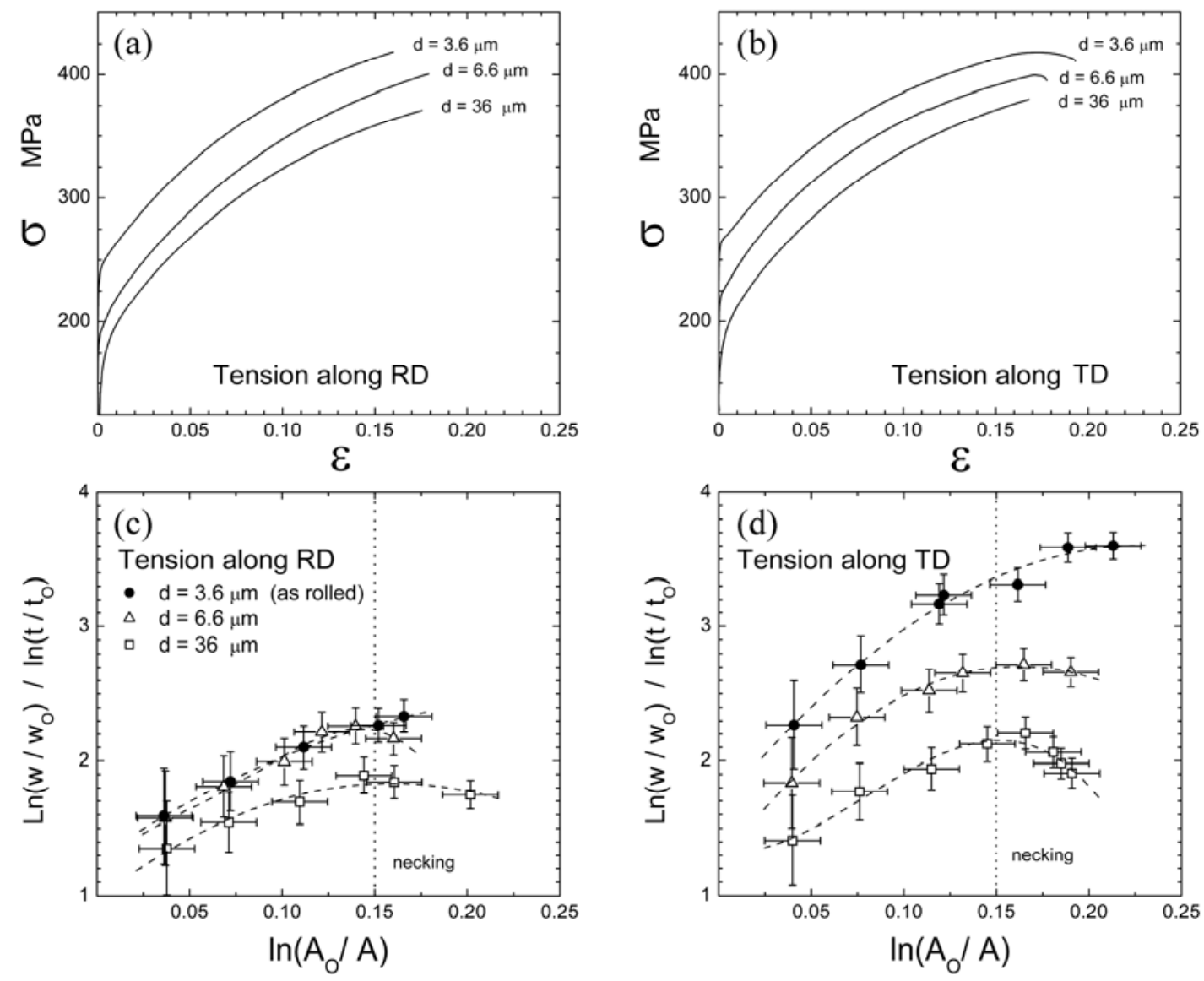

Figure 3. (a) and (b) stress-strain curves of RD and TD samples respectively. (c) and (d) evolution of the anisotropy as a function of plastic strain for RD and TD samples respectively.

\section{References}

[1] del Valle JA, Carreño F, Ruano OA. Acta Mater 2006; 54: 4247-59.

[2] Barnett MR, Keshavarz Z, Beer AG, Atwell D. Acta Mater 2004; 52: 5093-103

[3] Yang Q, Ghosh AK. Acta Mater 2006; 54: 5159-70

[4] Hwang S, Nishimura C, McCormick PG. Scripta Mater 2001; 44: 1507-11.

[5] del Valle JA, Ruano OA. Scripta Mater 2006; 55: 775-8.

[6] Barnett MR. Mat Trans 2003; 44: 571-7.

[7] Hauser FE, Landon PR, Dorn JE. Trans ASM 1955; 48: 986-1002.

[8] Koike J, Ohyama R, Kobayashi T, Suzuki M, Maruyama K. Mater Trans 2003; 44: 445-51.

[9] Jain A, Duygulu O, Brown DW, Tomé CN, Agnew SR. Mat Sci Eng 2008; A486: 545-55.

[10] Agnew SR, Duygulu O. Int J Plast 2005; 21: 1161-93.

[11] del Valle JA, Pérez-Prado MT, Ruano OA. Mat Sci Eng 2003; A355: 68-78.

[12] Roberts CS. The deformation of magnesium. Magnesium and Its Alloys. New York: Wiley; 1964.

[13] Proust G, Tomé CN, Jain A, Agnew SR. Int J Plast 2008, doi:10.1016/j.iplas.2008.05.005

[14] del Valle JA, Ruano OA. Acta Mater 2007; 55: 455-66.

[15] Armstrong RW. Acta Metall 1968; 16: 347-55. 